\title{
GH replacement causing acute hyperglycaemia and ketonuria in a type 1 diabetic patient
}

\author{
Dominic Cavlan, Shanti Vijayaraghavan', Susan Gelding' and William Drake \\ Department of Endocrinology, St Bartholomew's Hospital, West Smithfield, London, UK and \\ ${ }^{1}$ Newham University Hospital, Glen Road, London E13 8SL, UK
}

Correspondence

should be addressed

to D Cavlan

Email

dominic.cavlan@bartshealth. nhs.uk

\section{Summary}

A state of insulin resistance is common to the clinical conditions of both chronic growth hormone (GH) deficiency and GH excess (acromegaly). GH has a physiological role in glucose metabolism in the acute settings of fast and exercise and is the only anabolic hormone secreted in the fasting state. We report the case of a patient in whom knowledge of this aspect of $\mathrm{GH}$ physiology was vital to her care. A woman with well-controlled type 1 diabetes mellitus who developed hypopituitarism following the birth of her first child required GH replacement therapy. Hours after the first dose, she developed a rapid metabolic deterioration and awoke with hyperglycaemia and ketonuria. She adjusted her insulin dose accordingly, but the pattern was repeated with each subsequent increase in her dose. Acute GH-induced lipolysis results in an abundance of free fatty acids (FFA); these directly inhibit glucose uptake into muscle, and this can lead to hyperglycaemia. This glucose-fatty acid cycle was first described by Randle et al. in 1963; it is a nutrient-mediated fine control that allows oxidative muscle to switch between glucose and fatty acids as fuel, depending on their availability. We describe the mechanism in detail.

\section{Learning points:}

- There is a complex interplay between GH and insulin resistance: chronically, both GH excess and deficiency lead to insulin resistance, but there is also an acute mechanism that is less well appreciated by clinicians.

- GH activates hormone-sensitive lipase to release FFA into the circulation; these may inhibit the uptake of glucose leading to hyperglycaemia and ketosis in the type 1 diabetic patient.

- The Randle cycle, or glucose-fatty acid cycle, outlines the mechanism for this acute relationship.

- Monitoring the adequacy of GH replacement in patients with type 1 diabetes is difficult, with IGF1 an unreliable marker.

\section{Background}

This case illustrates the complex nature of the interactions between growth hormone (GH) and glucose metabolism and is a reminder of the potential hazards of GH replacement in patients with type 1 diabetes mellitus (T1DM). The acute effects of GH on insulin sensitivity are less well appreciated by clinicians than the long-term effects.

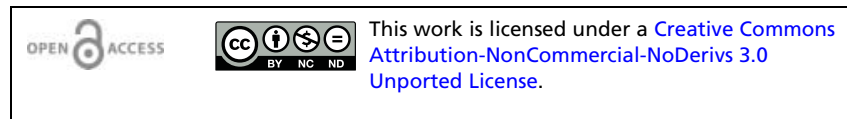

\section{Case presentation}

A 41-year-old Caucasian woman, diagnosed with T1DM as a child, underwent IVF therapy to conceive her first child. In addition, she was being treated with levothyroxine for autoimmune hypothyroidism, had suffered with endometriosis and had been given a label of chronic fatigue syndrome by her family physician. Her diabetes

$\begin{array}{rr}\text { (C) } 2013 \text { The authors } & \text { http://www.edmcasereports.com } \\ \text { Published by Bioscientifica Ltd }\end{array}$


was well-controlled prior to pregnancy on a basal bolus regimen, but she was converted to insulin pump therapy in the first trimester - by the third trimester, she was using 35-40 units of soluble insulin daily.

Labour was induced at 36 weeks due to polyhydramnios, but failure to progress and a pathological cardiotocogram (CTG) at full dilatation led to a caesarean section. This was complicated by a post partum haemorrhage of $1250 \mathrm{ml}$, which was managed with misoprostol, without recourse to blood transfusion. A female infant weighing $3.17 \mathrm{~kg}$ was delivered and spent a short time on the special care baby unit for treatment of pneumonia.

In the post-natal period, there was a precipitous decline in her health and vitality. She complained of fatigue, lassitude, increased anxiety and poor concentration and was finding it difficult to lose weight. She was unable to lactate, and menses did not return. She also consulted a gynaecologist with regard to dyspareunia. Her total insulin requirements dropped to 10 units/day with frequent hypoglycaemia, with a significant proportion nocturnal; pre-conception requirements had been 25-30 units daily.

Clinical examination was unremarkable other than for a resting pulse rate of 48 b.p.m., which rose appropriately with exertion. Blood pressure was $100 / 70 \mathrm{mmHg}$ without any postural drop. Visual fields were intact, and there was no evidence of any retinopathy. This was confirmed at retinal screening.

\section{Investigation}

Hormonal testing revealed an undetectable serum insulin-like growth factor (IGF1), luteinizing hormone $=$ $3.1 \mathrm{U} / \mathrm{ml}$, follicle-stimulating hormone $=7.1 \mathrm{U} / \mathrm{ml}$ and prolactin $=107 \mathrm{mU} / 1$ (125-625). Thyroid function on $100 \mu \mathrm{g}$ levothyroxine daily was normal $(\mathrm{TSH}=1.37 \mathrm{mIU} / 1$ $(0.3-4.2)$, free thyroxine $=17.3 \mathrm{pmol} / 1$ (9.0-26.0)). A $0900 \mathrm{~h}$ cortisol was $365 \mathrm{nmol} / \mathrm{l}$. Magnetic resonance imaging scan of the pituitary gland revealed no abnormalities and she underwent dynamic testing to assess cortisol and GH reserve with an insulin tolerance test. The results can be seen in Table 1. Her cortisol reserve was satisfactory, but the peak GH level after adequate hypoglycaemia was $0.195 \mu \mathrm{g} / \mathrm{l}$, confirming biochemically severe GH deficiency (GHD). Her score on the Adult Growth Hormone Deficiency Assessment (AGHDA) questionnaire was $18 / 25$, and GH replacement therapy was recommended, at a starting dose of $0.1 \mathrm{mg}$ daily.
Table 1 Insulin tolerance test.

\begin{tabular}{|c|c|c|c|}
\hline Time (min) & $\begin{array}{l}\text { Glucose } \\
(\mathrm{mmol} / \mathrm{l})\end{array}$ & $\begin{array}{l}\text { Cortisol } \\
\text { (nmol/l) }\end{array}$ & $\begin{array}{c}\text { Growth } \\
\text { hormone }(\mu \mathrm{g} / \mathrm{l})\end{array}$ \\
\hline 0 & 12.4 & 462 & 0.259 \\
\hline 30 & 3.21 & 354 & 0.171 \\
\hline 45 & 1.86 & 384 & 0.229 \\
\hline 60 & 1.59 & 579 & 0.195 \\
\hline 75 & 1.48 & 728 & 0.153 \\
\hline 90 & 1.55 & 846 & 0.135 \\
\hline 120 & 3.1 & 786 & 0.107 \\
\hline 150 & 4.3 & 759 & 0.154 \\
\hline
\end{tabular}

\section{Treatment}

The patient awoke a few hours after the first dose of GH with nausea and capillary blood glucose of $19.0 \mathrm{mmol} / \mathrm{l}$, and her urine was positive for ketones on dipstick testing. This was the first such episode since her childhood. She was administered a bolus dose of soluble insulin using her pump and immediately increased the basal rate. Over the subsequent days, she established a new equilibrium of 25 units insulin daily by continuous s.c. infusion. Her GH dose was titrated very gradually over several months up to $0.4 \mathrm{mg}$ daily, but she remained prone to ketonuria in the early morning and with a few days of altered glycaemic control at each new dose. Her HbA1c exhibited a deterioration from 7.1 to $7.6 \%$ over this time course.

Prior to GH replacement, she received oral oestrogen and progesterone replacement. This was converted to a transdermal preparation comprising oestradiol hemihydrate $75 \mu \mathrm{g} / 24 \mathrm{~h}$ for 2 weeks, followed by oestradiol hemihydrate $50 \mu \mathrm{g} / 24 \mathrm{~h}$ and norethisterone acetate $170 \mu \mathrm{g} / 24 \mathrm{~h}$. The change from oral to transdermal oestrogen replacement brought about a further slight increase in her insulin requirements, along with nausea and malaise that were intolerable to her. She therefore returned to oral replacement with conjugated oestrogens and norgestrel and has established separate basal rates of insulin infusion according to hormone replacement: 8.7 units insulin aspart daily on oestrogen only; 9.0 units daily on combined oestrogen and progesterone.

\section{Outcome and follow-up}

Monitoring adequacy of GH replacement using IGF1 was not straightforward as she failed to establish IGF1 in the upper half of her age-related reference range despite improvement in her symptoms. A decision was taken to make dose adjustments based on clinical grounds, 
given that her diabetes mellitus would make IGF1 levels more difficult to interpret than in a non-diabetic patient. She has gained a significant clinical benefit from GH replacement, with an AGHDA score of 9/25 after 2 years of GH replacement.

\section{Discussion}

This patient's previously tightly controlled T1DM was decompensated within hours by a single dose of $0.1 \mathrm{mg}$ $\mathrm{GH}$. Given the reproducible effects of subsequent $0.1 \mathrm{mg}$ dose increases, this is highly unlikely to have been a coincidental phenomenon, and it raises the issue of the complex relationship between GH and insulin sensitivity.

Isolated GHD may present with hypoglycaemia, but both GH excess and long-term adult GHD are insulinresistant states (1). T2DM develops in around 15\% of patients with acromegaly, and adolescent individuals are more insulin resistant than either adults or pre-pubertal children. GHD becomes a more insulin-resistant state the longer it persists, possibly as a consequence of increased visceral adiposity (2). A significant body of evidence has emerged since the first trials of recombinant human GH replacement in 1989, that it is associated with increases in lean body mass and a reduction in central adiposity, and that it leads to improvements in HbA1c (3). Such longterm effects cannot provide the mechanism for the extremely rapid development of ketotic hyperglycaemia in our patient.

Abrupt GH-mediated derangement of glycaemic control was demonstrated as long ago as 1958, when Luft et al. (4) used cadaveric GH to treat three type 1 diabetic patients with hypopituitarism and induced immediate hyperglycaemia and ketonuria. Randle et al. (5) hypothesised that GH caused liberation of free fatty acids (FFA), which themselves have a direct effect on increasing insulin resistance. FFA release from isolated rat tissues was increased by prior starvation or by treatment with steroid or GH and was decreased by hypophysectomy, or by treatment with insulin. It was also demonstrated that infusion of FFA or ketone bodies would increase insulin resistance in rat skeletal muscle.

It was by then well established that the ratio of insulin to glucagon had a role in determining whether lipid was stored as adipose tissue or released from it, but Randle et al. (5) added a nutrient-mediated fine control on top of the hormonal control, highlighting the ability of oxidative muscle to switch between fuels depending on their availability. Where FFA are more abundant, glucose uptake and oxidation would be inhibited, while conversely FFA oxidation would be inhibited by high levels of glucose. The mechanisms for these effects have been established (6) and are illustrated in Fig. 1.

In T1DM, there is a close correlation between levels of GH and FFA, both physiologically and pharmacologically. Administration of a single dose of GH causes a marked increase in both serum FFA and ketones and induces insulin resistance by $2 \mathrm{~h}$ (7). Co-administration of an inhibitor of hormone-sensitive lipase with $\mathrm{GH}$, to block FFA release, leads to no change in insulin sensitivity (8). When GH secretion is inhibited by somatostatin blockade, infusion of FFA alone leads to a dose-dependent increase in insulin resistance (9).

It seems likely that our patient's rapid metabolic deterioration was due to acute GH-induced lipolysis and was responsible for the sudden increase in insulin resistance, but in the longer term, the same lipolysis serves to improve insulin sensitivity by breaking down stores of visceral fat, in concert with the GH-associated improvement in well-being and increased physical activity (1).

A further issue brought to light by this case is the use of serum IGF1 in the biochemical assessment of type 1 diabetic patients on GH replacement. These patients have

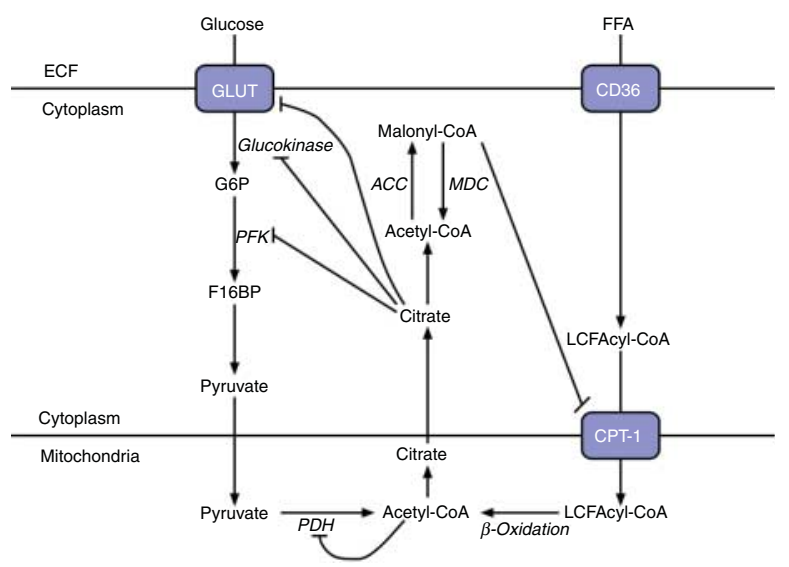

Figure 1

The glucose-fatty acid cycle. A simplified diagram showing the interactions of products of glucose metabolism with free fatty acids (FFA) uptake and vice versa. ACC, acetyl-CoA carboxylase; F16BP, fructose 1,6 bisphosphate; G6P, glucose 6 phosphate; $\mathrm{PDH}$, pyruvate dehydrogenase; PFK, phosphofructokinase; LCFAcyl CoA, long-chain fatty acyl CoA; MDC, malonyl-CoA decarboxylase; ECF, extracellular fluid. Malonyl-CoA is a by-product of glucose oxidation and inhibits carnitine palmitoyl transferase, an enzyme controlling fatty acid entry into the mitochondria. Malonyl-CoA concentrations in the steady state are a balance between the activity of ACC and MDC. When FFA is more abundant, metabolites favour activity of $M C D$ via inhibition of the protein kinase A signalling pathway. When glucose is in excess, this pathway is stimulated, malonyl-CoA levels increase and FFA metabolism is inhibited. 
higher and more fluctuant levels of GH and lower levels of IGF1, probably related to a lack of portal vein insulin, and resultant down-regulation of hepatic GH receptors (10). Using IGF1 as a marker of adequacy of GH replacement is therefore problematic; one approach is to titrate the $\mathrm{GH}$ dose to an IGF1 between the age-related median and the upper limit of normal, but in T1DM this is not possible as was evident in this patient. We aim to titrate to adequate clinical response without side effects in such patients.

In conclusion, this case illustrates some of the complexities inherent in $\mathrm{GH}$ replacement in a patient with type 1 diabetes. We wish to remind colleagues of the potential hazards of commencing $\mathrm{GH}$ replacement in these individuals.

\section{Declaration of interest}

The authors declare that there is no conflict of interest that could be perceived as prejudicing the impartiality of the research reported.

\section{Funding}

This research did not receive any specific grant from any funding agency in the public, commercial or not-for-profit sector.

\section{Patient consent}

Written informed consent has been obtained from the patient for publication of the case report.

\section{Author contribution statement}

W Drake is the patient's consultant endocrinologist; D Cavlan saw the patient regularly in the outpatient clinic and wrote the manuscript.

\section{References}

1 Svensson J \& Bengtsson B-A 2003 Growth hormone replacement therapy and insulin sensitivity. Journal of Clinical Endocrinology and Metabolism 88 1453-1454. (doi:10.1210/jc.2003-030218)

2 Møller N \& Jørgensen JO 2009 Effects of growth hormone on glucose, lipid, and protein metabolism in human subjects. Endocrine Reviews 30 152-177. (doi:10.1210/er.2008-0027)

3 Gotherstrom G, Bengtsson BA, Bosaeus I, Johannsson G \& Svensson J 2007 A 10-year prospective study of the metabolic effects of growth hormone replacement in adults. Journal of Clinical Endocrinology and Metabolism 92 1442-1445. (doi:10.1210/jc.2006-1487)

4 Luft R, Ikkos D, Gemzell CA \& Olivecrona H 1958 Effect of human growth hormone in hypophysectomised diabetic subjects. Lancet $\mathbf{1}$ 721-722. (doi:10.1016/S0140-6736(58)91142-5)

5 Randle PJ, Garland PB, Hales CN \& Newsholme EA 1963 The glucose fatty-acid cycle. Its role in insulin sensitivity and the metabolic disturbances of diabetes mellitus. Lancet 1 785-789. (doi:10.1016/ S0140-6736(63)91500-9)

6 Hue L \& Taegtmeyer H 2009 The Randle cycle revisited: a new head for an old hat. American Journal of Physiology. Endocrinology and Metabolism 297 E578-E591. (doi:10.1152/ajpendo.00093.2009)

7 Møller N, Jørgensen JO, Schmitz O, Møller J, Christiansen J, Alberti KG \& Orskov H 1990 Effects of a growth hormone pulse on total and forearm substrate fluxes in humans. American Journal of Physiology $\mathbf{2 5 8}$ E86-E91.

8 Nielsen S, Møller N, Christiansen JS \& Jørgensen JO 2001 Pharmacological antilipolysis restores insulin sensitivity during growth hormone exposure. Diabetes 50 2301-2308. (doi:10.2337/diabetes.50. 10.2301)

9 Gormsen LC, Jessen N, Gjedsted J, Gjedde S, Norrelund H, Lund S, Christiansen JS, Nielsen S, Schmitz O \& Møller N 2007 Dose-response effects of free fatty acids on glucose and lipid metabolism during somatostatin blockade of growth hormone and insulin in humans. Journal of Clinical Endocrinology and Metabolism 92 1834-1842. (doi:10.1210/jc.2006-2659)

10 Christ ER, Simpson HL, Breen L, Sönksen PH, Russell-Jones DL \& Kohner EM 2003 The effect of growth hormone (GH) replacement therapy in adult patients with type 1 diabetes mellitus and GH deficiency. Clinical Endocrinology 58 309-315. (doi:10.1046/ j.1365-2265.2003.01714.x)

Received in final form 31 July 2013

Accepted 11 September 2013 